\title{
On-Line Gas Chemistry Experiments with Transactinide Elements
}

\author{
H.W. Gäggeler \\ Paul Scherrer Institut \\ $\mathrm{CH}-5232$ Villigen \\ Switzerland
}

\section{Introduction}

Investigations of the chemical properties of heaviest elements is of great current interest, the reason being relativistic effects which should play an important role in determining their chemical properties. Several recent theoretical studies revealed that due to the high charge of the nuclei the spherical $7 \mathrm{~s}$ or $6 \mathrm{p}_{1 / 2}$ orbitals are stabilized whereas the high angular momentum $6 \mathrm{~d}$ or $5 \mathrm{f}$ orbitals are destabilized [1]. As an examples, it was concluded that the last element of the actinides series, lawrencium, might behave more like a p-rather than a d-element [1]. Several experiments which tried to confirm this prediction failed, however $[2,3]$. Also for the elements 104 (rutherfordium) and 105 (hahnium) deviations in their chemical behaviours from those expected on the basis of extrapolations within the groups of the periodic table are expected. As an example, a much higher volatility of $\mathrm{HaBr}_{5}$ is predicted if compared to those of $\mathrm{TaBr}_{5}$ and $\mathrm{NbBr}_{5}$ [4]. An extrapolation based on the trend in group $\mathrm{V}$ would predict rather similar volatilities of $\mathrm{HaBr}_{5}, \mathrm{TaBr}_{5}$ and $\mathrm{NbBr}_{5}[5]$.

Another reason for the great current interest in heaviest elements stems from a predicted new subisland of nuclear stability around $Z=108$ and $N=162$. In the centre of this subisland spontaneous-fission half-lives of about $108 \mathrm{~s}$ and alpha half lives on the order of seconds are predicted [6]. These rather long half-lives give optimism for future chemistry experiments with elements above the presentday heaviest element hahnium, with which chemical studies has been performed so far. 


\section{On-line isothermal gas chromatography with the OLGA II and HEVI set ups}

Fast gas chemical separations have proven to be very successful in transactinide studies. Very early experiments made by Zvara et al. used the thermochromatography technique for investigations of rutherfordium chloride [7]. As a result it could be shown that the first transactinide element forms highly volatile halides, in contrast to actinides. This is expected on the basis of the position of transactinide elements in the periodic table, where rutherfordium belongs to group IV, hahnium to group $\mathrm{V}$ of the transition elements.

In this contribution, another technique will be discussed, the on-line isothermal gas chromatography in quartz columns. This technique was used in recent years to study the retention times of rutherfordium and hahnium chlorides and bromides. Two devices have been developed, OLGA II (On-Line Gas chemistry Apparatus, second version) [8] and HEVI (Heavy Element Volatility Instrument) [9]. OLGA I was a previous device for gas chromatographic separations without the possibility of using reactive gases. It was mainly used to search for volatile superheavy elements [10]. Fig. 1 depicts a general layout of the OLGA II and HEVI devices: reaction products are transported with a gas jet system from the place of production (i.e. a target chamber at an accelerator) to the gas chemistry apparatus. In such a system the products are attached to small aerosol particles (e.g. $\mathrm{KCl}, \mathrm{MoO}_{3}$ or graphite particles of about 50 to $200 \mathrm{~nm}$ size), transported through a thin capillary and then continuously injected into a quartz column. Here the aerosol particles containing the reaction products are collelcted on a quartz wool plug. This collector is heated to about 900 to $1000{ }^{\circ} \mathrm{C}$. Reactive gases are added to that position in order to form volatile halide molecules. Depending on the temperature of a second isothermal part of the chromatography column halide molecules are transported through the tube according to their volatility. Behind the column ejecting molecules are reattached to new particles in a socalled recluster unit. This procedure allows to transport the separated species along a capillary to a counting device. Two different counting units were used, a rotating wheel [9] or a stepwise moving tape system. [8]. Both systems are equipped with PIPS (passivated implanted planar silicon) detectors to measure alpha and spontaneous-fission decay. In case of chemical studies with gamma emitting nuclides, the aerosol behind the recluster unit was pumped through a glass fibre filter mounted on top of a HPGe detector. 
The total time needed for the separation of volatile molecules amounts to about $20 \mathrm{~s}$. This rather long time is caused by the recluster process. The OLGA II and HEVI techniques can therefore be applied to nuclides with $>10 \mathrm{~s}$ half-lives only. Maximum chemical yields for species having half-lives of $\geq 1 \mathrm{~min}$ are about $60 \%$.

\section{Volatilities of group IV and V halides}

As a measure of the volatility we use the vapour pressures of macroamounts of the liquid components, Figs. 2 and 3 show the vapour pressures of the fluorides, chlorides, bromides and iodides of $\mathrm{Zr}, \mathrm{Nb}, \mathrm{Hf}$, and $\mathrm{Ta}$ [11]. For group IV we find a volatility sequence $\mathrm{Cl}>\mathrm{Br}>\mathrm{I}>\mathrm{F}$ and $\mathrm{Hf}(\mathrm{hal})>\mathrm{Zr}$ (hal); (hal = halides). For group $\mathrm{V}$ the sequence is $\mathrm{F}>\mathrm{Cl}>\mathrm{Br}>\mathrm{I}$ (not shown in Fig. 3), and $\mathrm{Ta}$ (hal) $>\mathrm{Nb}$ (hal).

Figs. 2 and 3 show that group $\mathrm{V}$ chlorides are more volatile than group IV chlorides. For the bromides the volatilities of all mentioned elements are very similar.

In on-line experiments trace amounts of oxygen or water vapour may enter the gas system via diffusion through the walls of the transportation capillary. This can favour the formation of oxohalides instead of the pure halides. For the group IV and $\mathrm{V}$ elements the oxohalides are less volatile than the pure halides. For illustration, Fig. 4 (upper right part) shows the volatilities of $\mathrm{NbCl}_{5}$ and $\mathrm{NbOCl}_{3}$ [12].

Several experiments were performed with carrier-free tracers of $\mathrm{Zr}, \mathrm{Nb}, \mathrm{Hf}$, and $\mathrm{Ta}$, in order to learn how measurements of chromatographic curves obtained with the OLGA II or HEVI devices can be related to volatilities of macroamounts. Short-lived isotopes of $\mathrm{Zr}$ and $\mathrm{Nb}$ (e.g. $99 \mathrm{gNb}$ with $\mathrm{T}_{1 / 2}=15 \mathrm{~s}$ or $100 \mathrm{Zr}$ with $\mathrm{T}_{1 / 2}=$ $7.1 \mathrm{~s}$ ) were produced at the SAPHIR reactor at PSI. Hf and Ta isotopes (e.g. 162Hf with $\mathrm{T}_{1 / 2}=38 \mathrm{~s}$ or $167 \mathrm{Ta}$ with $\mathrm{T}_{1 / 2}=2.9 \mathrm{~min}$ ) were produced via alpha or heavy ion induced fusion reactions with the 88-inch cyclotron at the Lawrence Berkeley Laboratory, the UNILAC accelerator at the Gesellschaft für Schwerionenforschung in Darmstadt or the PHILIPS cyclotron at the Paul Scherrer Institut in Villigen.

Fig. 4 shows one example, where the yields of $99 \mathrm{gNb}$ are shown using $\mathrm{HCl}$ as chlorinating agent. The two curves were measured with and without purification of the carrier gas $(\mathrm{He})$ from oxygen. Purification was made by passing the $\mathrm{He}$ gas through a charcoal trap heated to $1000^{\circ} \mathrm{C}$. This reduced the oxygen content from about $100 \mathrm{ppm}$ to less than $1 \mathrm{ppm}$. From an intercomparison of the measured 
chromatograms with the volatility curves of the macrocomponents (see upper right part in Fig. 4) it can be concluded that the $99 \mathrm{gNb}$ species which reaches maximum yields at $\mathrm{T} \geq 200{ }^{\circ} \mathrm{C}$ is most likely $\mathrm{NbCl}_{5}$, whereas that one which reaches the maximum yield at $\mathrm{T} \geq 300{ }^{\circ} \mathrm{C}$ is $\mathrm{NbOCl}_{3}$.

In general, these studies revealed that the temperature difference between the measured chromatographic slopes of two species is nearly identical to the difference in the temperature between the corresponding vapour pressure curves (e.g. about $100^{\circ} \mathrm{C}$ in Fig. 4).

\section{On-line isothermal gas chromatography experiments with rutherfordium and hahnium}

The nuclides $261 \mathrm{Rf}\left(\mathrm{T}_{1 / 2}=78 \mathrm{~s}\right)$ and $262.263 \mathrm{Ha}\left(\mathrm{T}_{1 / 2}=30 \mathrm{~s}\right)$ were produced in the $248 \mathrm{Cm}(18 \mathrm{O}, 5 \mathrm{n})$ and the $249 \mathrm{Bk}(18 \mathrm{O}, 4,5 \mathrm{n})$ reactions, respectively, at the 88 -Inch cyclotron at LBL and at the UNILAC accelerator at GSI. The targets contained 500 to $900 \mu \mathrm{g} / \mathrm{cm}^{2}$ of $248 \mathrm{Cm}$ or $249 \mathrm{Bk}$, electrodeposited on a Be foil. To these targets typical beam currents of $0.5 \mathrm{p} \mu \mathrm{A}$ were applied. For transportation of the reaction products a $\mathrm{He}$ carrier gas was used which contained $\mathrm{KCl}$ or $\mathrm{MoO}_{3}$ particles.

Fig. 5 shows a $\alpha$ spectrum obtained in a Rf chloride experiment [13]. As reactive gas $100 \mathrm{ml} / \mathrm{min}$ of $\mathrm{HCl}$ was added to the $\mathrm{He} / \mathrm{MoO}_{3}$ carrier aerosol. In the energy range 8.18 to $8.38 \mathrm{MeV}$ a total of $837 \alpha$-particles could be attributed to the decay of $261 \mathrm{Rf}$ and its daughter $257 \mathrm{No}$, as was confirmed by a decay analysis. Besides this peak several other lines from Po and $\mathrm{Bi}$ isotopes are visible. These elements also form very volatile halides and can therefore not be separated from transactinide halides. Since these elements are most likely produced in nucleon transfer reactions between the projectile and $\mathrm{Pb}$ and/or $\mathrm{Bi}$ impurities in the target, the "contamination" of the $\alpha$-spectrum by these lines depends strongly on the purity of the target. To achieve an unambiguous assignment of the measured events to single atoms of transactinide elements it is therefore very important to register the $\alpha$ and fission events in a list mode. This allows a detection of correlated events. As an example, Fig. 6 depicts correlated $\alpha$ - events which were measured in a gas chemistry experiment with hahnium chloride [14]. As reactive gas $100 \mathrm{ml} / \mathrm{min}$ of $\mathrm{Cl}_{2}$ saturated by $\mathrm{CCl}_{4}$ vapour was used. On the basis of the measured life times (i.e. half-lives) of the mother $(262 \mathrm{Ha})$ and the daughter $(258 \mathrm{Lr})$ nuclides and of both $\alpha$ energies, each observed decaying atom can be unambiguously assigned to hahnium. 
In some cases, the detection of spontaneous-fission events was used for the determination of chemical yields. It is well known that in nucleon transfer reactions many spontaneously fissioning nuclides are formed. This makes an unambiguous assignment to one specific isotope very difficult. One example, where spontaneous-fission was used for a yield determination was a study of hahnium bromide, using the $50 \%$ sf-branch of $262 \mathrm{Ha}$ [15]. In this case a careful decay analysis was made to confirm this assignment.

Fig. 7 shows the measured relative (maximum yield $=100 \%$ ) chemical yields of $98 \mathrm{Zr}, 162 \mathrm{Hf}$ and $261 \mathrm{Rf}$ using chlorinating gases and $\mathrm{MoO}_{3}$ as transporting particles [19]. The curves for $\mathrm{Rf}$ and $\mathrm{Zr}$ chlorides are very similar. Surprisingly, the curve for $162 \mathrm{Hf}$ is shifted towards higher temperatures. This is not expected on the basis of the vapour pressure curves (see Fig. 2). Also shown in Fig. 7 are the adsorption enthalpies as deduced from the measured curves. They were obtained with a Monte Carlo model developed by Zvara [17] and applied to isothermal gas chromatography by Tuerler [18]. In Fig. 8 we have summarized adsorption enthalpies for $\mathrm{Zr}$, Hf, and of $\mathrm{Rf}$ chlorides from this work and from the literature [19]. Most of the literature data were obtained by the thermochromatography technique. We clearly observe a grouping of all existing data around two distinct adsorption enthalpies, 70 and $90 \mathrm{~kJ} / \mathrm{mol}$. We assign the $70 \mathrm{~kJ} / \mathrm{mol}$ values to the pure tetra halides. Whether the species with $90 \mathrm{~kJ} / \mathrm{mol}$ adsorption enthalpy resemble oxohalides is unclear; another possibility would be a transport in the chromatographic tube via heterogeneous surface reactions, so-called transport reactions [20].

Also shown in Fig. 8 is the adsorption enthalpy given for rutherfordium chloride by Zvara et al. [7]. The rather high adsorption enthalpy would fit better to a oxochloride (or a transport reaction) than to a pure chloride. The data depicted in Fig. 8 clearly show that within experimental resolution no trend in the adsorption enthalpies (and, hence, no trend in the volatilities) of the group IV chlorides of $\mathrm{Zr}$, $\mathrm{Hf}$ and $\mathrm{Rf}$ can be observed.

For rutherfordium bromide, one preliminary experiment was performed with a $\mathrm{He} / \mathrm{KCl}$ gas jet and $100 \mathrm{ml} / \mathrm{min} \mathrm{HBr}$ as reactive gas [13]. As a result, the chromatographic curve was shifted towards higher temperatures if compared to that of rutherfordium chloride indicating a lower volatility. However, it is well known, that with salt particles such as $\mathrm{KCl}$ the surface of the quartz column might become covered by this material during the experiment. This can then cause an increase in the retention temperature due to surface reactions with this 
salt [15]. Future experiments with rutherfordium bromide are therefore needed with particles such as $\mathrm{MoO}_{3}$ or graphite. $\mathrm{MoO}_{3}$ forms very volatile oxohalides which do not deposit in quartz columns except for very low temperatures $\leqslant 150$ ${ }^{\circ} \mathrm{C}$ ). Graphite is oxidised by trace amounts of water vapour and oxygen in the carrier gas to gaseous $\mathrm{CO}$ and $\mathrm{CO}_{2}$. The $\mathrm{MoO}_{3}$ and graphite particle containing gas jet systems therefore allow chromatographic studies on pure quartz surfaces.

Fig. 9 shows the measured chromatograms of $\mathrm{Nb}, \mathrm{Ta}$ and $\mathrm{Ha}$ bromides from different experiments, all using a $\mathrm{He} / \mathrm{KCl}$ gas jet [15,21]. The curve for $\mathrm{Nb}$ was measured with pure $\mathrm{HBr}$, for Ta with $\mathrm{HBr}$ containing $\mathrm{BBr}_{3}$ vapour, and for $\mathrm{Ha}$ with both, pure $\mathrm{HBr}$ as well as $\mathrm{HBr}$ containing $\mathrm{BBr}_{3}$ vapour. Fig. 8 shows that the curves for $\mathrm{Nb}$ and $\mathrm{Ta}$ are nearly identical, as expected on the basis of their vapour pressures (see Fig. 3). The curve of hahnium bromide is shifted towards higher temperatures. Also shown in the figure are deduced adsorption enthalpies. The higher adsorption enthalpy for hahnium bromide if compared to those of the homologous niobium and tantalum bromides would indicate a lower volatility. Fig. 10 shows the estimated vapour pressure curve for $\mathrm{HaBr}_{5}$, calculated on the basis of an empirical correlation between adsorption enthalpies of trace amounts of bromides on quartz surfaces and the sublimation enthalpies of the corresponding macroamounts [5,22]. Also shown in Fig. 10 are the theoretically calculated vapour pressure curves for $\mathrm{HaBr}_{5}$, based on a relativistic molecular calculation [4] (theor.-rel.) as well as on a semi-empirical thermodynamical model [5] (theor.-non-rel.). The relativistic model would predict a higher volatility of $\mathrm{HaBr}_{5}$ if compared to those of $\mathrm{NbBr}_{5}$ and $\mathrm{TaBr}_{5}$, whereas the "non-relativistic" thermodynamical model predicts nearly equal volatilities for all group $\mathrm{V}$ pentabromides. Both predictions clearly deviate from the experimental results. At present it has to be assumed that eather hahnium bromide is indeed less volatile than the corresponing bromides of the group V elements or (more likely) with the gas system used in the experimental study it was not possible to form the pure pentabromide of hahnium but rather the oxobromide. Additional experiments are needed with $\mathrm{MoO}_{3}$ or graphite particles as well as with chlorinating gases.

\section{Outlook to future on-line isothermal gas chemistry experiments}

Both set-ups OLGA II and HEVI suffer from two shortcomings, a very long average separation time of about $20 \mathrm{~s}$ and a poor chromatographic resolution, the latter being caused by the short chromatography column length in the isothermal section of the oven (only about 10 to $20 \mathrm{~cm}$ ). We have therefore developed a new 
device, called OLGA III, based on a commercially available gas chromatography oven (Carlo Erba; maximum temperature $=500^{\circ} \mathrm{C}$ ) [23]. Such an oven system enables a temperature stabilization which is superior to a tubular oven and the use of much longer ( $>1 \mathrm{~m}$ ) columns. The column is coiled around a support and connected to a small $(200 \mathrm{ml})$ water cooled recluster unit. It could be shown that OLGA III performs chemistry separations within about two seconds [23]. Due to the longer columns, the chromatographic resolution could be improved considerably. With this new device it will be possible to perform high quality retention time measurements in order to investigate minor changes in the volatilities of halides of elements belonging to one group of the periodic table. Moreover, due to the short separation times of OLGA III future studies with neutron-rich isotopes of the elements 106, 107 (nielsbohrium) and 108 (hassium) seem to be possible.

\section{References}

[1] see e.g.O.L.Keller, Radiochim. Acta, 37, 169 (1984)

[2] U.W. Scherer et al., Inorg. Chim. Acta, 146, 249(1988)

[3] D.T.Jost et al., Inorg. Chim. Acta, 146, 255(1988)

[4] V.Pershina et al., J. Chem.Phys., 97,1116 (1992)

[5] B.Eichler, Progress report 1993, Annex III, Paul Scherrer Insttitut, Villigen, Switzerland, in press (1994)

[6] see e.g.. A. Sobiczewski, Proc. 6th Int. Conf. on Nuclei far from Stability, Bernkastel-Kues, 19-24 July 1992; Inst. Phys. Conf. Ser. No 132:Section 4

[7] see e.g. I. Zvara et al., J. Inorg. Nucl.Chem., 32, 1885(1970)

[8] H.W. Gāggeler et al., Nucl. Instr. Meth., A309, 201(1991)

[9] B.Kadkhodayan et al., Nucl. Instr.Meth., A317, 254(1992)

[10] P. Armbruster et al., Phys. Rev.Lett., 54, 406(1985)

[11] from A.I. Efimov, "Svoistva neorganicekikh soedinenii Izd. Khimija", Leningrad (1983)

[12] V.Pershina et al., J. Chem. Phys. 97 1123(1992)

[13] A. Türler et al., J. Radioanal. Nucl. Chem.,160, 327(1992) 
[14] A. Türler et al., priv. communication (1993), and to be published

[15] H.W. Gäggeler et al., Radiochim. Acta, 57, 93(1992)

[16] B. Kadkhodayan et al., to be submitted to Radiochim. Acta (1994)

[17] I. Zvara, Radiochim. Acta, 38, 95(1985)

[18] A. Tuerler, Progress Report 1992, Paul Scherrer Institut, Annex III, Villigen, Switzerland, p. 95 (1993), and to be published

[19] A. Türler, Proc. School Seminar on Heavy Ion Physics, Dubna, May 1993, in print (1994)

[20] B. Eichler et al., Radiochim. Acta, 61, 81(1993)

[21] Ya Nai-Qi et al., Radiochim. Acta, 47, 1(1989)

[22] U. Kim et al., Isotopenpraxis 24, 30(1988)

[23] R. Eichler et al., Progress Report 1992, Paul Scherrer Institut, Annex III, Villigen Switzerland, p. 99 (1993) 


\section{Figure captions}

Fig. 1: Schematic of the on-line gas chemistry set-ups OLGA II [8] and HEVI.[9]. Reaction products are injected with a gas jet to a quartz wool plug which is heated to about $1000^{\circ} \mathrm{C}$. To this position reactive gases such as halogenating compounds can be added. Volatile species are then transported along the quartz column which is kept at an isothermal temperature. Behind the column the molecules are ejected into a recluster unit where they are attached to new aerosol particles and then transported to a counting device. This device is a stepwise moving tape system or a rotating wheel. Final products are assayed for $\alpha$ decay or spontaneous fission using heavy ion implanted silicon detectors.

Fig. 2: Vapour pressure curves for zircon and hafnium chlorides, bromides, iodides and fluorides [11].

Fig. 3: Vapour pressure curves for niobium and tantalum chlorides, bromides and fluorides [11].

Fig. 4: Gas chemistry yields for niobium chlorides, produced with $15 \mathrm{~s}-99 \mathrm{gNb}$, at two different partial pressures of oxygen in the gas system. For chlorination $\mathrm{HCl}$ gas and for transportation helium gas containing graphite particles was used. Also shown in the upper right corner are the vapour pressure curves of $\mathrm{NbCl}_{5}$ and of $\mathrm{NbOCl}_{3}$, respectively.

Fig. 5: $\alpha$ spectrum of a gas chemistry experiment to study to the chlorides produced in the reaction $18 \mathrm{O}+{ }^{248} \mathrm{Cm}$ (from [13]).

Fig. 6: Correlated $\alpha-\alpha$ events from a gas chemistry experiment to study the chlorides from the reaction ${ }^{18} \mathrm{O}+{ }^{249} \mathrm{Bk}$ (from [14]). Every decaying nuclide is characterized by four signals: $\alpha$ energies of mother and daughter isotopes as well

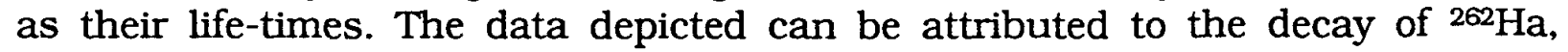
except for the two events with $\alpha$ - energies $<8.3 \mathrm{MeV}$ of the mother. We attribute these two events to ${ }^{261} \mathrm{Rf}$.

Fig. 7: Gas chemistry yields for zirconium, hafnium and rutherfordium chlorides. Inserted in the figure are the chlorinating gases used and the deduced adsorption enthalpies (from [16,19]).

Fig. 8: Adsorption enthalpies of chlorides of zirconium, hafnium and rutherfordium from isothermal chromatography and thermochromatography experiments (see text).

Fig. 9: Gas chemistry yields for niobium, tantalum and hahnium bromides. Inserted in the figure are the brominating gases used and the deduced adsorption enthalpies (from [15]).

Fig. 10: Vapour pressure curves for niobium, tantalum and hahnium bromides. The curves for niobium and tantalum are from [11]. The curve $\mathrm{HaBr}_{5}$ (exp.) is deduced from the experimental data shown in Fig. 9 (see text). The curve $\mathrm{HaBr}_{5}$ (theor.-rel.) is from [4] and the area denoted $\mathrm{HaBr}_{5}$ (theor.-non-rel.) from [5], respectively. 


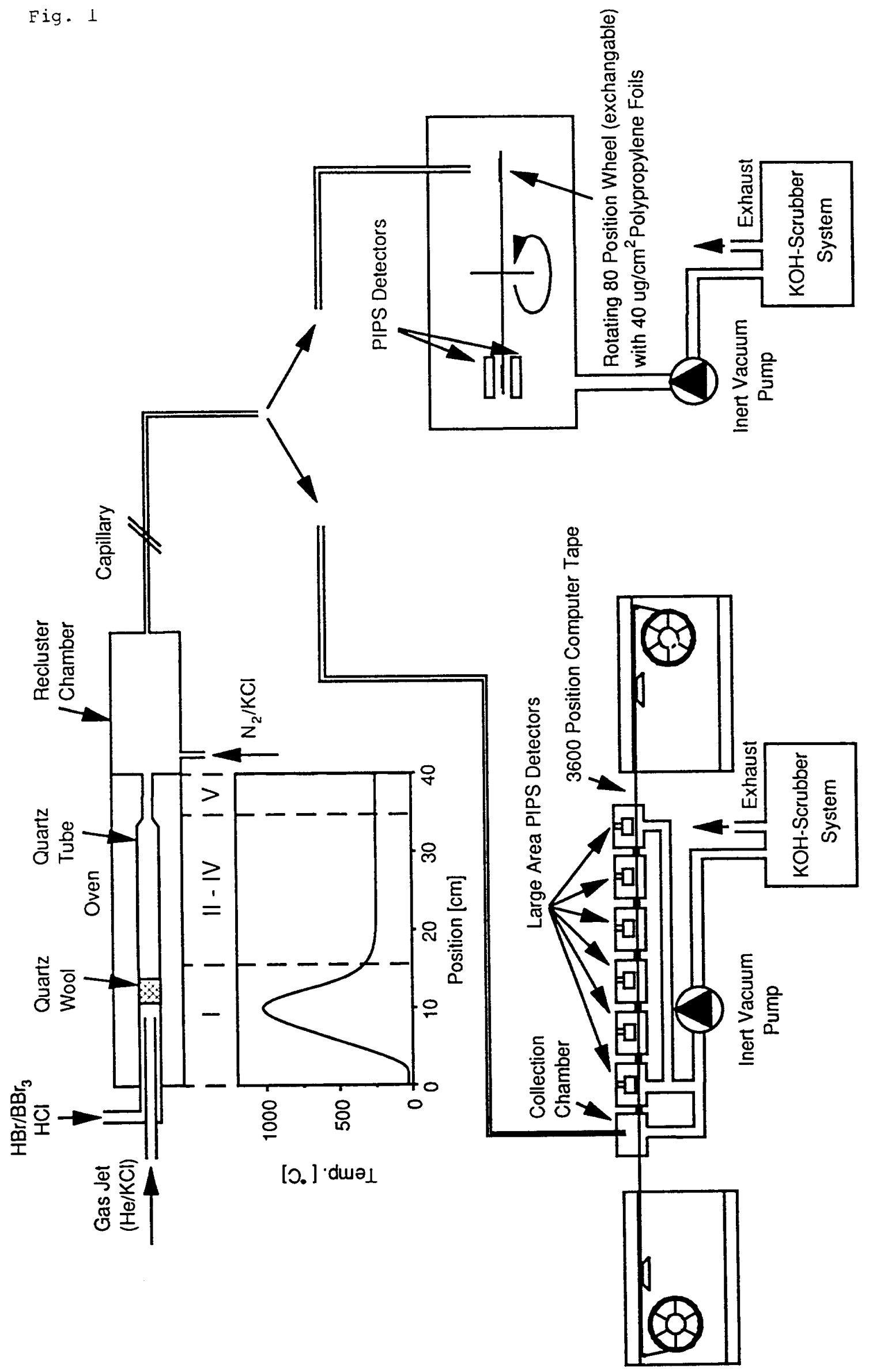


Fig. 2

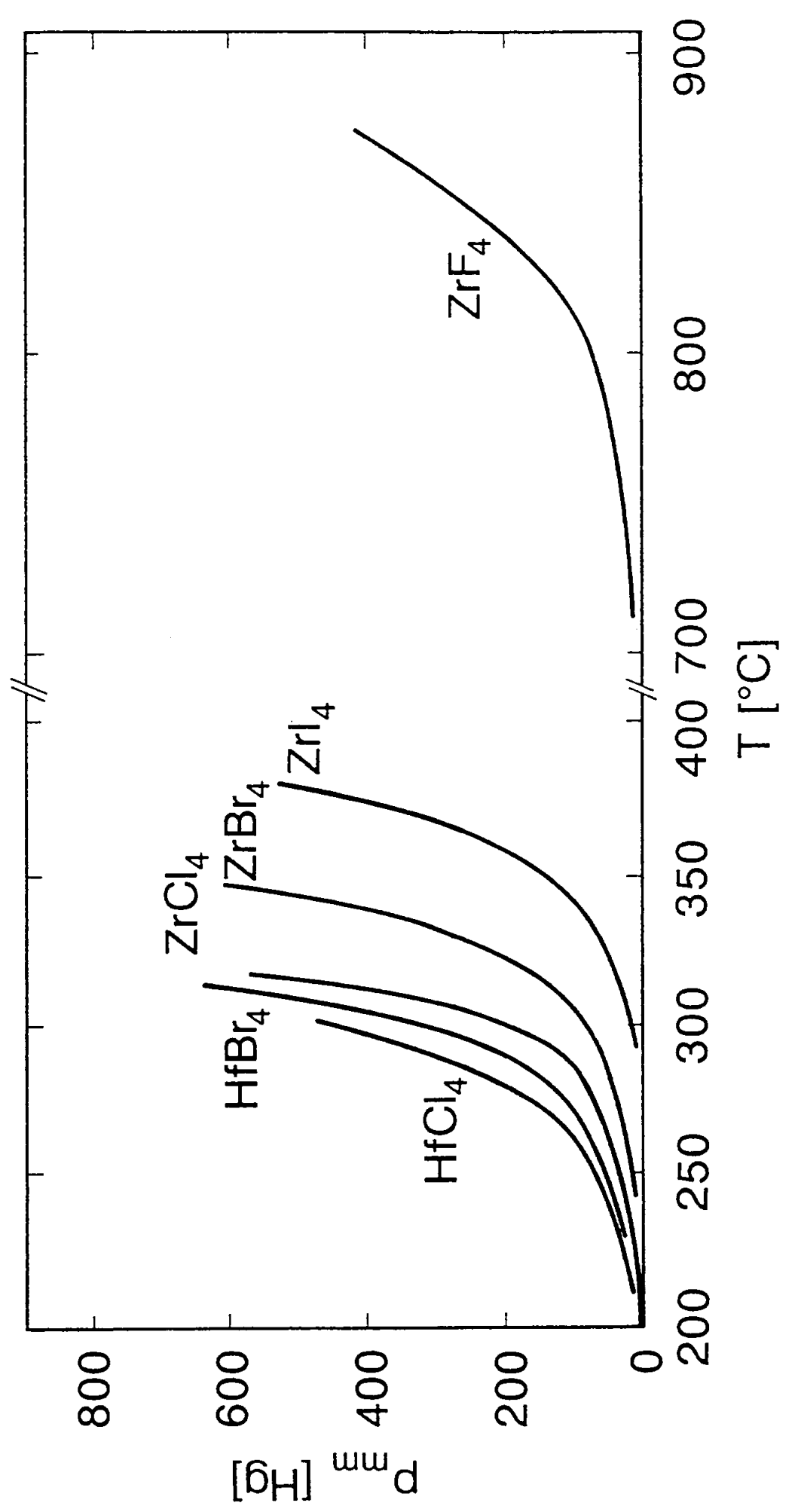


Fig. 3

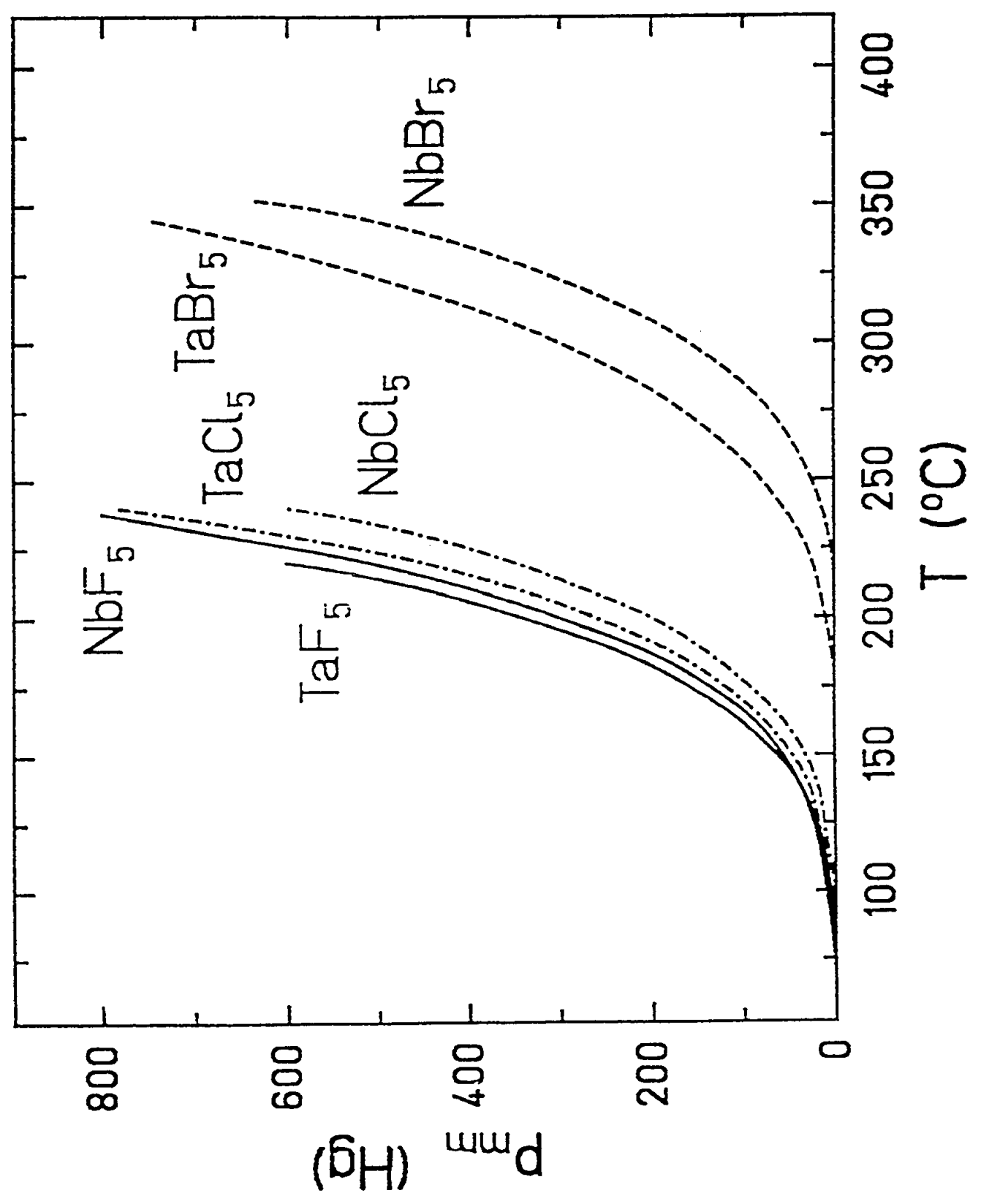


Fig. 4

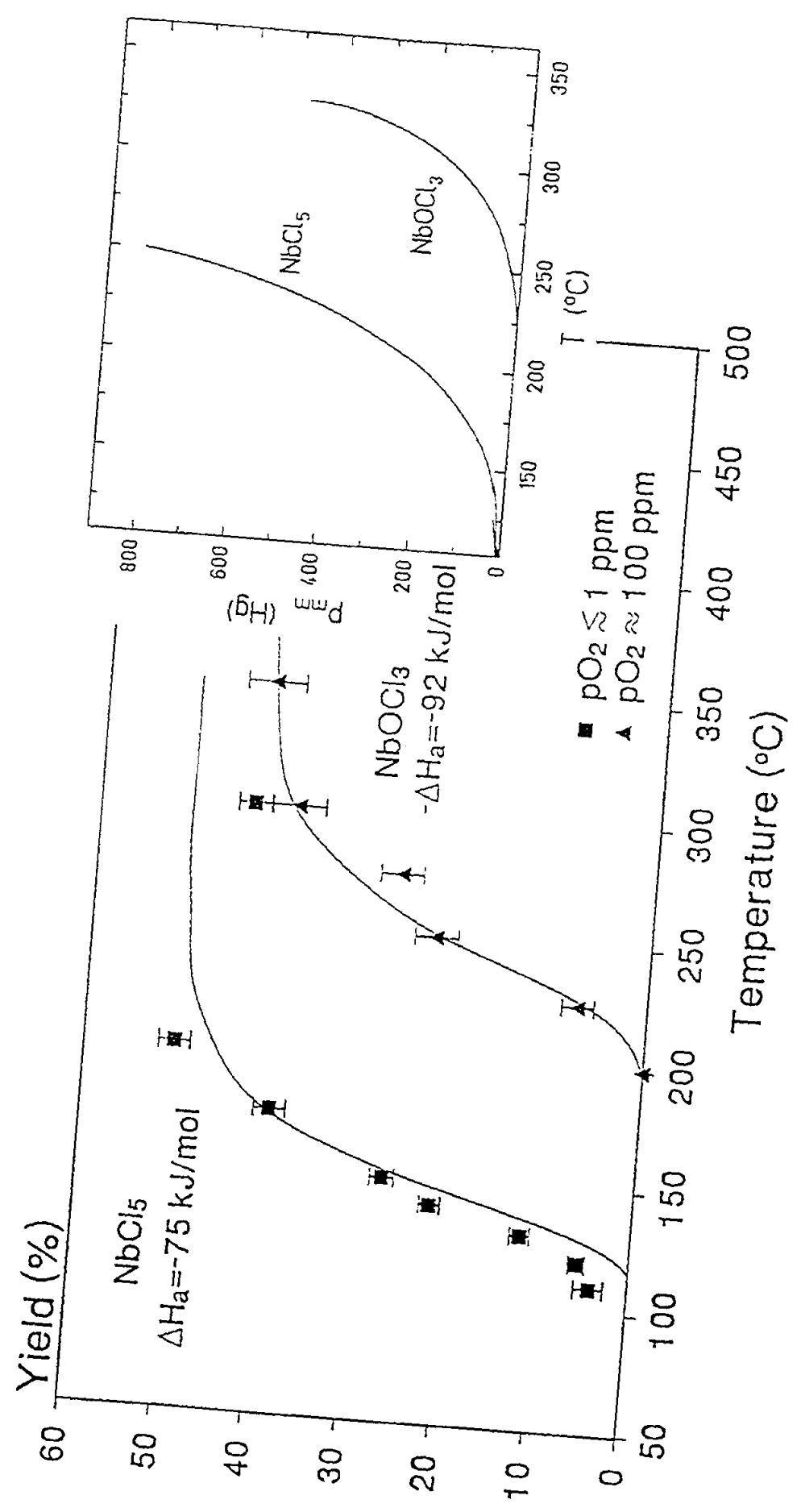


Fig. 5

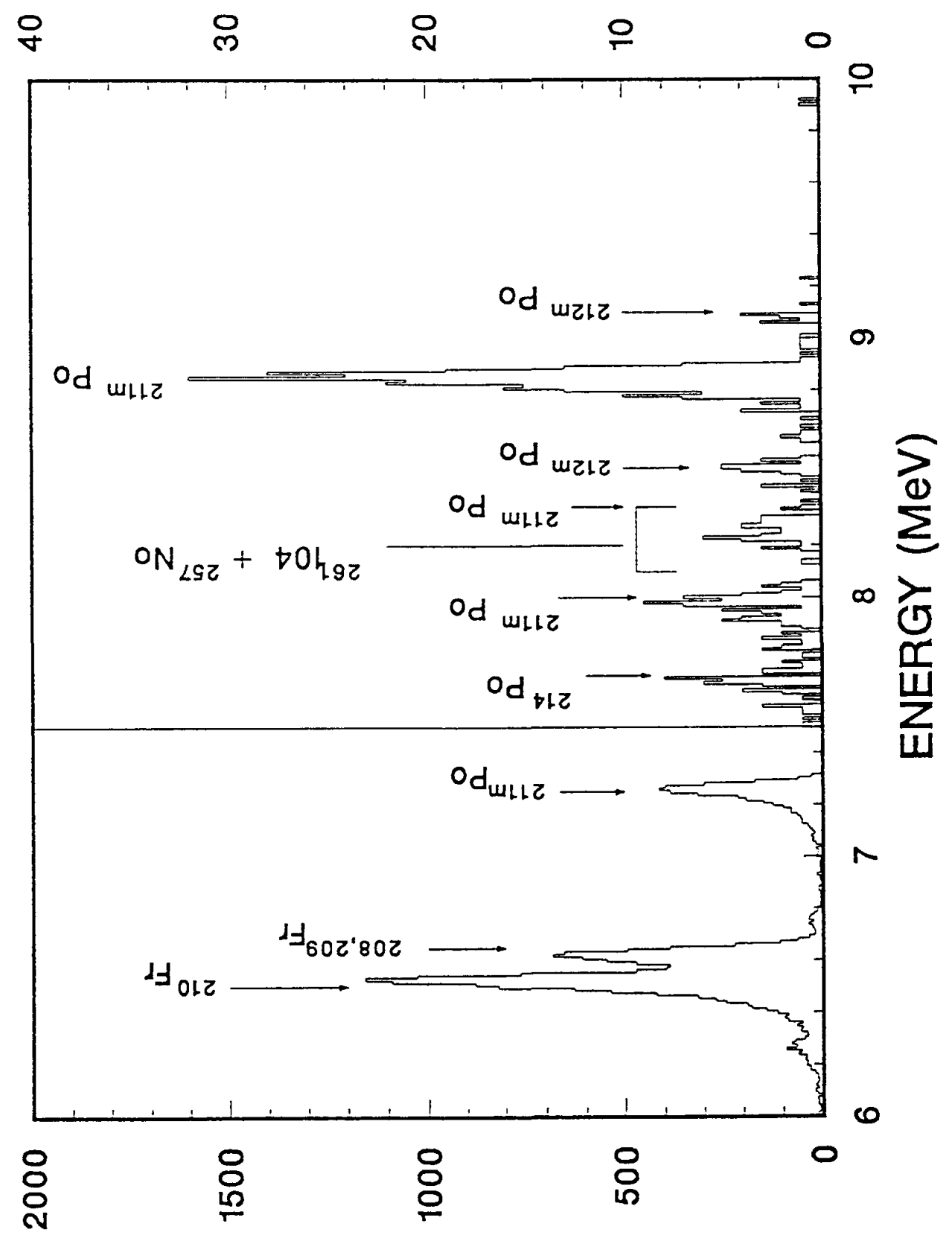

$\operatorname{S\perp N} \exists \wedge \exists$ 
Fig. 6

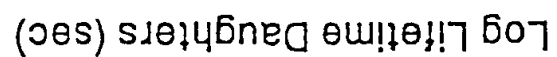

0.2 0.1 0.0 0.1- $0^{\circ} \mathrm{Z}-$

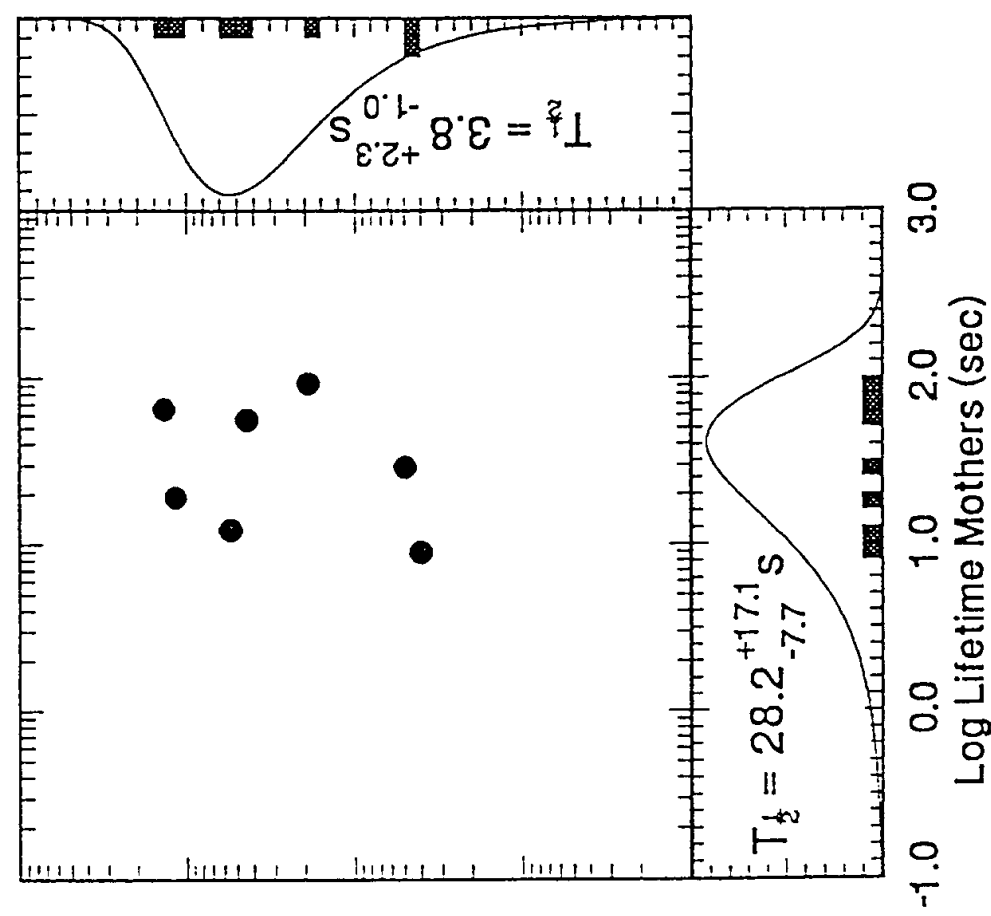

$5, x$ seutbinea

$\begin{array}{llllll}00 \angle 8 & 0098 & 0098 & 0078 & 0088 & 0028\end{array}$

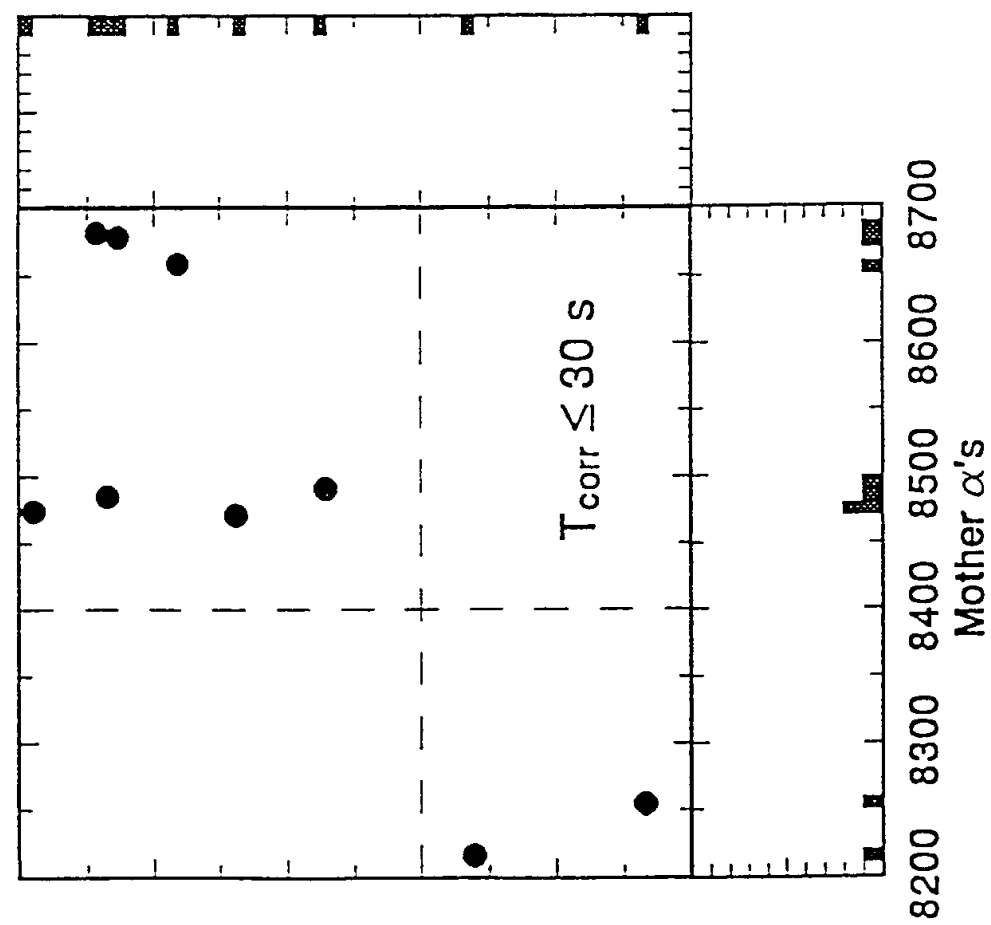




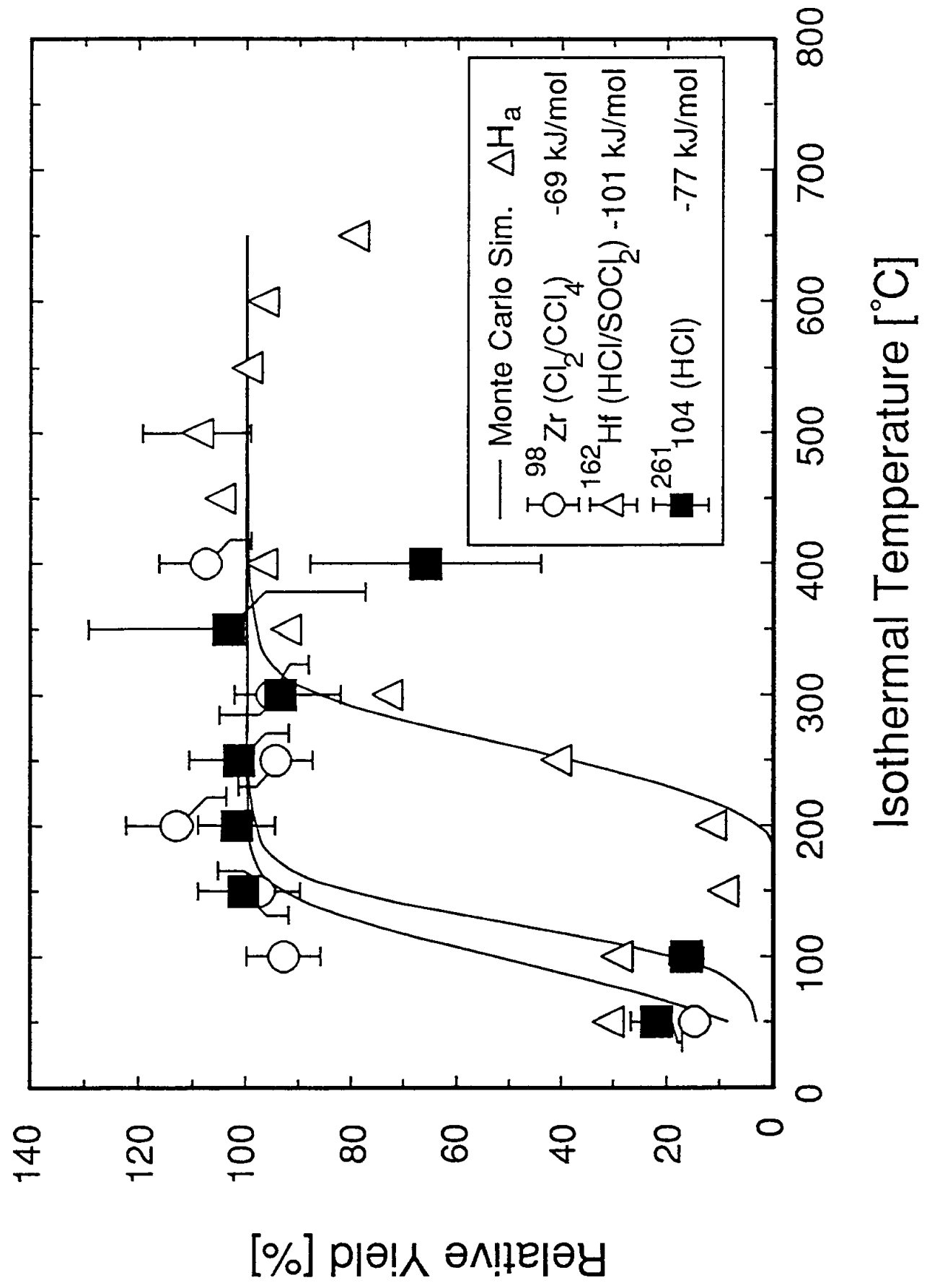


Fig. 8

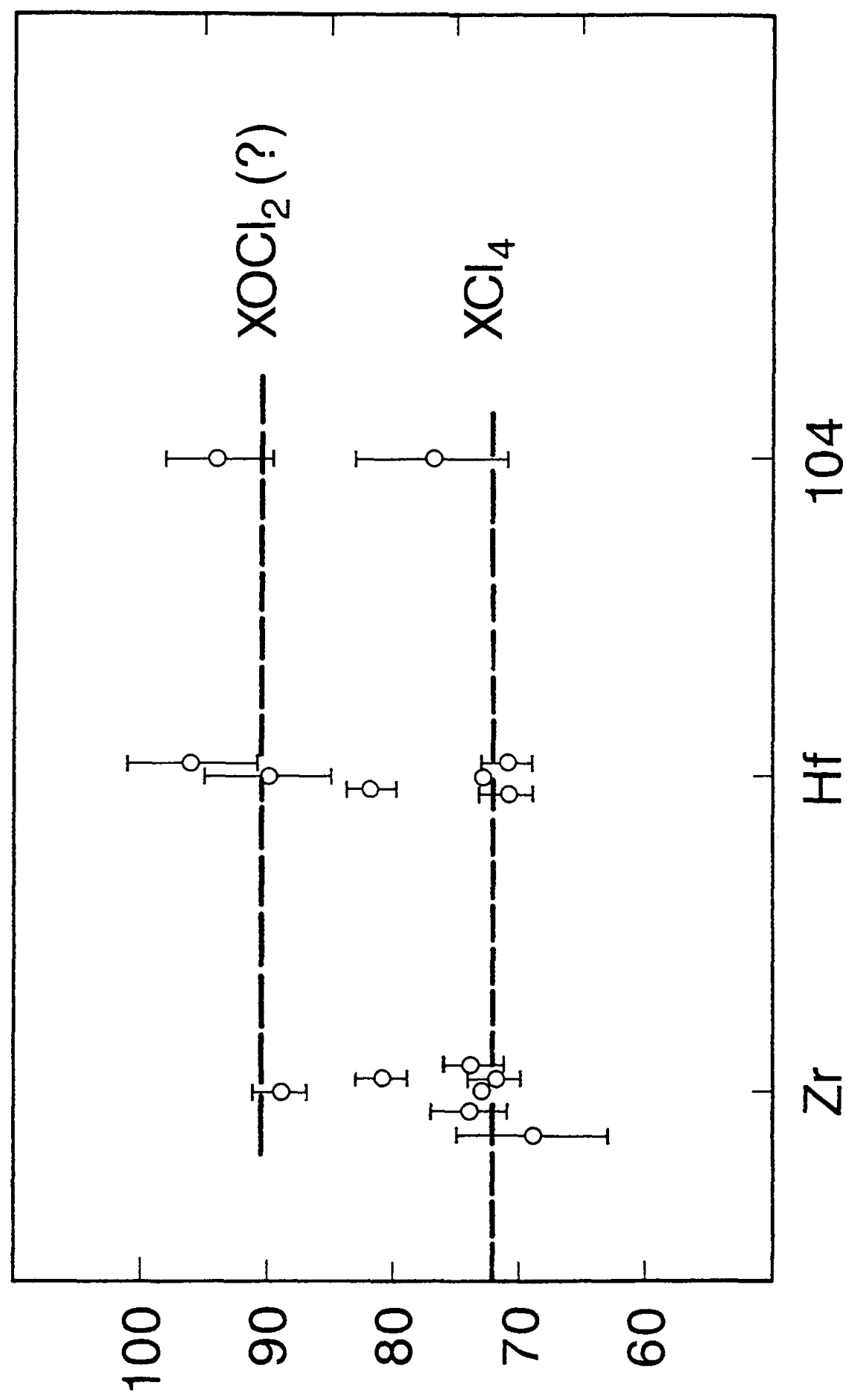

[jom/Гr] Кdןeułuə uo!̣dıosp $\forall$ 
Fig. 9

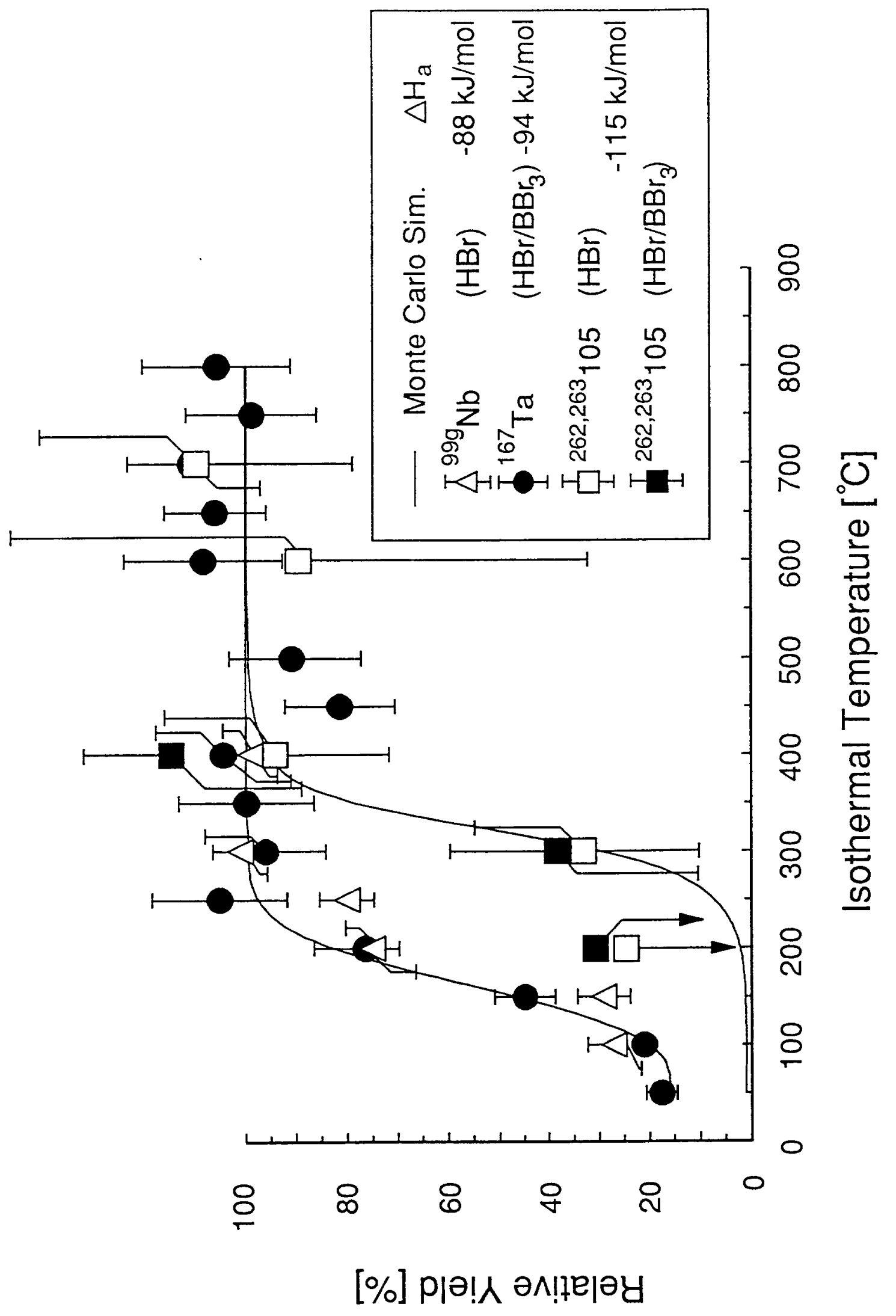




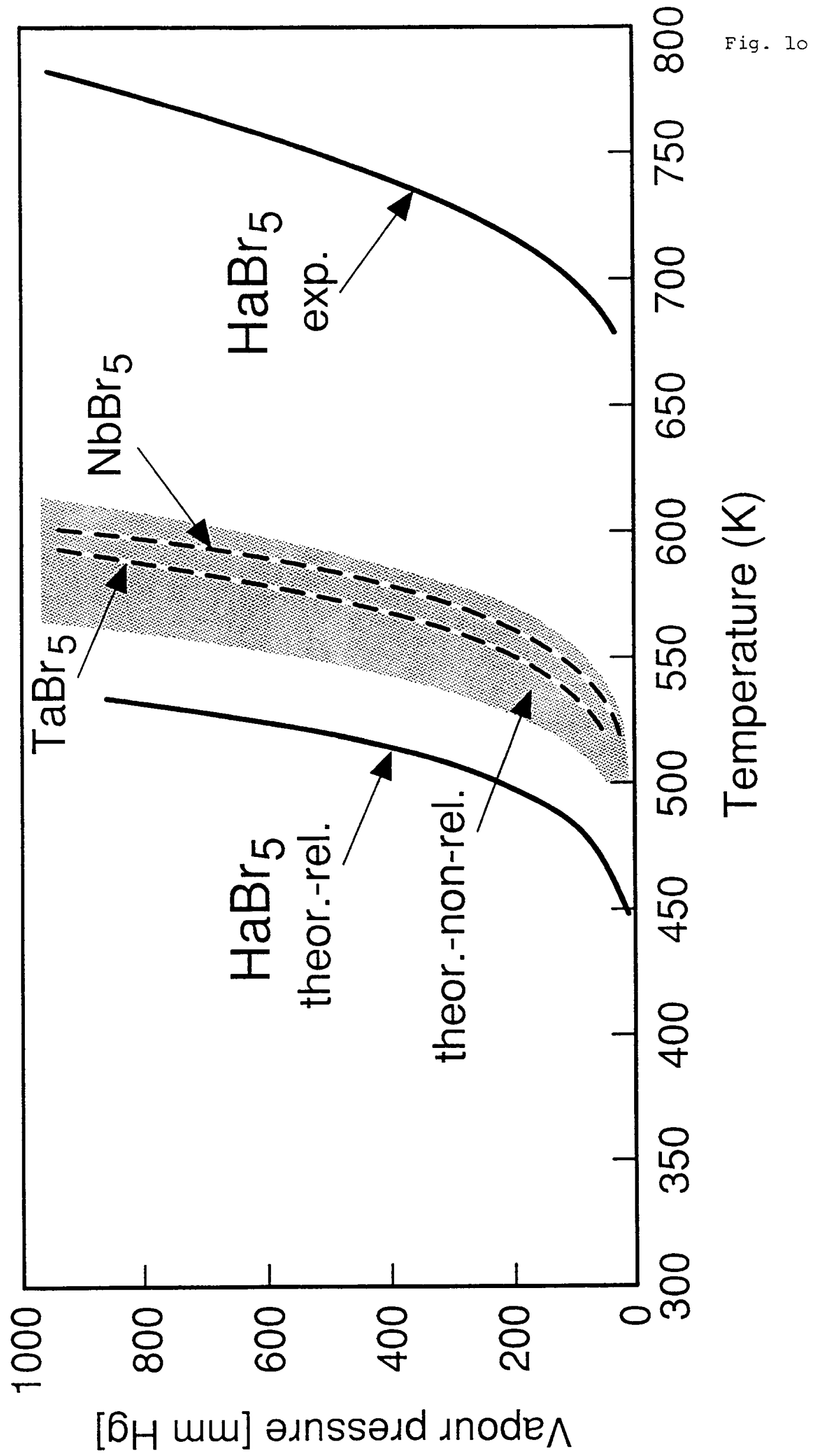


